\title{
Erratum: Endoscopic Features of Upper Gastrointestinal Tract in Patients with Systemic Sclerosis Compared to the Healthy Control
}

Jun Won Park ${ }^{1}$, Jihye Kim ${ }^{2,3}$, Eun Ae Kang ${ }^{3}$, Min Jung Kim ${ }^{1}$, Joo Sung Kim³ ${ }^{3}$ Eun Bong Lee

${ }^{1}$ Division of Rheumatology, Department of Internal Medicine, Seoul National University College of Medicine, ${ }^{2}$ Department of Internal Medicine, CHA Gangnam Medical Center, CHA University School of Medicine, ${ }^{3}$ Department of Internal Medicine and Liver Research Institute, Seoul National University College of Medicine, Seoul, Korea

J Rheum Dis 2019;26:66-73

https://doi.org/10.4078/jrd.2019.26.1.66

The original version of this article contained an error of the acknowledgement (funding source).

The correct acknowledgement (funding source) is as follows:

This research was supported by a grant of the Korea Health Technology R\&D Project through the Korea Health Industry Development Institute (KHIDI), funded by the Ministry of Health \& Welfare, Republic of Korea (grant number: HC17C0069).

The funding source has been already revised in the journal's online site.

We apologize for this error. 